\title{
Brecha digital y Covid-19: percepciones y dificultades. Un estudio en tres instituciones educativas de Antioquia
}

Carlos Arango*, Diana Castaño**, Katerine Cuervo Botero***, Yus Deisy Baena ****

DOI: https://doi.org/:10.33571/revistaluciernaga.v12n24a7

\section{Resumen}

Este estudio presenta la forma como tres instituciones educativas experimentaron la brecha digital en el escenario de la cuarentena vivida en Colombia motivada por el Covid-19 y que afectó la modalidad presencial en los procesos educativos. Se abordó una etnografía digital, la cual permitió el acercamiento a las percepciones que la educación en ambientes alternativos de aprendizaje generó en estudiantes, docentes y padres de familia. Luego de una exploración del concepto brecha digital, se exponen los resultados, donde se evidencian los múltiples niveles en que opera la brecha, a partir de las percepciones de los grupos sociales abordados en el estudio. A lo largo del texto se aportan evidencias para sostener que la brecha digital no es otra cosa que la manifestación en otros entornos (de aprendizaje, en este caso) de la profunda brecha social que viven muchos ciudadanos del mundo contemporáneo.

Palabras clave: TIC; brecha digital; ambientes alternativos de aprendizaje; educación; conectividad; comunidad educativa; Covid-19.

Recibido. Mayo 28, $2020 \quad$ Aceptado. Junio 09, 2020

*Comunicador, Magíster y Doctorando en Filosofía. Líder grupo de investigación Communis, programa de Comunicación Social, Universidad Católica de Oriente. Áreas de trabajo: imaginarios, música, consumo. Autor principal. Orientó la metodología, supervisó los instrumentos de investigación, redacción y edición de la versión final. Orcid: https://orcid. org/0000-0002-2120-3304; e-mail: carango@uco.edu.co carango@uco.edu.co

**Especialista en Pedagogía y Didáctica. Licenciada en Educación Básica Primaria. Experiencia en docencia en educación primaria. Participó en la discusión de la pregunta, la metodología, el trabajo de campo y el análisis de resultados. Aplicación de instrumentos y estado del arte. E-mail: dicala129@gmail.com

***Especialista en Pedagogía y Didáctica. Licenciada en Educación Básica con énfasis en humanidades y lengua castellana. Experiencia en Docencia en educación primaria. Participó en la discusión de la pregunta, la metodología, el trabajo de campo y el análisis de resultados. Aplicación de instrumentos y estado del arte. E-mail: cuboka@gmail.com

${ }^{* * * \star}$ Especialista en Pedagogía y Didáctica. Licenciada en educación básica con énfasis en matemáticas. Experiencia en Docencia en educación primaria y secundaria. Participó en la discusión de la pregunta, la metodología, el trabajo de campo y el análisis de resultados. Aplicación de instrumentos y estado del arte. E-mail: deibary2275@gmail.com 


\title{
Digital gap and Covid-19: perceptions and constraints. A study in three educational institutions in Antioquia
}

\author{
Carlos Arango*, Diana Castaño**, Katerine Cuervo Botero ***, Yus Deisy Baena **** \\ DOI: https://doi.org/:10.33571/revistaluciernaga.v12n24a7
}

\begin{abstract}
This study presents how three educational institutions have experienced the digital gap in the scenario of the quarantine experimented in Colombia due to Covid-19, which affected the face-to-face modality in the educational processes, a digital ethnography was approached, which allowed the perception that education in alternative learning environments aroused in students, teachers, and parents. After an exploration of digital gap concept, the results showed the multiple levels this gap operates, based on the perceptions of the social groups addressed in the study. Throughout the text, evidence, it is argued that the digital gap is nothing more than the manifestation in other environments (learning, in this case) of the deep social gap experienced by a number of citizens of the contemporary world.
\end{abstract}

Keywords: ICT; digital gap; alternative learning environments; education; connectivity; educational community; Covid-19.

Received. May 28, $2020 \quad$ Accepted. June 09, 2020

*Communicator, Master and PhD student in Philosophy. Leader of the Communis research group, Social Communication program, Universidad Católica de Oriente. Areas of work: imaginaries, music, consumption. Main author. Methodological guidance, supervision of research instruments, writing and editing of the final version. Orcid: https://orcid.org/0000-00022120-3304; e-mail: carango@uco.edu.co

**Specialist in Pedagogy and Didactics. Bachelor's degree in Elementary Education. Teaching experience in primary education. Participation in the discussion of the question, methodology, field work and analysis of results. Implementation of instruments and state of the art. E-mail: dicala129@gmail.com

***Specialist in Pedagogy and Didactics. Bachelor's degree in Basic Education with emphasis in humanities and Spanish language. Teaching experience in elementary education. Participation in the discussion of the question, methodology, field work and analysis of results. Implementation of instruments and state of the art. E-mail: cuboka@gmail.com

****Specialist in Pedagogy and Didactics. Bachelor's degree in basic education with emphasis in mathematics. Teaching experience in primary and secondary education. Participation in the discussion of the question, methodology, field work and analysis of results. Implementation of instruments and state of the art. E-mail: deibary2275@gmail.com 


\section{Brecha digital e Covid-19: percepções e dificuldades. Um estudo em três instituições de ensino em Antioquia \\ Carlos Arango*, Diana Castaño**, Katerine Cuervo Botero ***, Yus Deisy Baena ***}

DOI: https://doi.org/:10.33571/revistaluciernaga.v12n24a7

\section{Resumo}

Este estudo apresenta a forma como três instituições educativas experimentaram a brecha digital no cenário da quarentena vivida na Colômbia motivada pelo Covid-19 e que afectou a modalidade presencial nos processos educativos. Foi abordada uma etnografia digital, a qual permitiu a abordagem das percepções que a educação em ambientes de aprendizagem alternativos gerou nos estudantes, professores e pais de famílias. Após uma exploração do conceito de brecha digital, são apresentados os resultados, onde são evidenciados os múltiplos níveis em que a brecha opera, a partir das percepções dos grupos sociais abordados no estudo. Ao longo do texto, são fornecidas evidências para argumentar que a brecha digital não é outra coisa que a manifestação, noutros ambientes (de aprendizagem, neste caso), da profunda brecha social que vivem muitos cidadãos do mundo contemporâneo.

Palavras chave: TIC; brecha digital; ambientes alternativos de aprendizagem ; educação; conectividade; comunidade educativa; Covid-19.

Recebido. Maio 28, 2020. Aceitado. Junho 09, 2020

${ }^{*}$ Comunicador, estudante de mestrado e doutoramento em Filosofia. Líder do grupo de investigação Communis, programa de Comunicação Social, Universidade Católica de Oriente. Áreas de trabalho: imaginários, música, consumo. Orcid: https:// orcid.org/0000-0002-2120-3304 ; e-mail: carango@uco.edu.co ; autor principal. Orientação metodológica, supervisão dos instrumentos de investigação, redacção e edição da versão final.

**Especialista em Pedagogia e Didáctica. Bacharelato em Ensino Elementar. Experiência de ensino no ensino primário. E-mail: dicala129@gmail.com; participação na discussão da questão, metodologia, trabalho de campo e análise dos resultados. Aplicação de instrumentos e estado da arte.

${ }^{* * * E s p e c i a l i s t a ~ e m ~ P e d a g o g i a ~ e ~ D i d a ́ c t i c a . ~ L i c e n c i a t u r a ~ e m ~ E d u c a c ̧ a ̃ o ~ B a ́ s i c a ~ c o m ~ e ̂ n f a s e ~ e m ~ h u m a n i d a d e s ~ e ~ l i ́ n g u a ~}$ espanhola. Experiência de ensino no ensino primário. E-mail: cuboka@gmail.com; participação na discussão da questão, metodologia, trabalho de campo e análise dos resultados. Aplicação de instrumentos e estado da arte.

****Especialista em Pedagogia e Didáctica. Bacharelato em educação básica com ênfase em matemática. Experiência docente no ensino primário e secundário. E-mail: deibary2275@gmail.com ; participação na discussão da questão, metodologia, trabalho de campo e análise dos resultados. Aplicação de instrumentos e estado da arte.

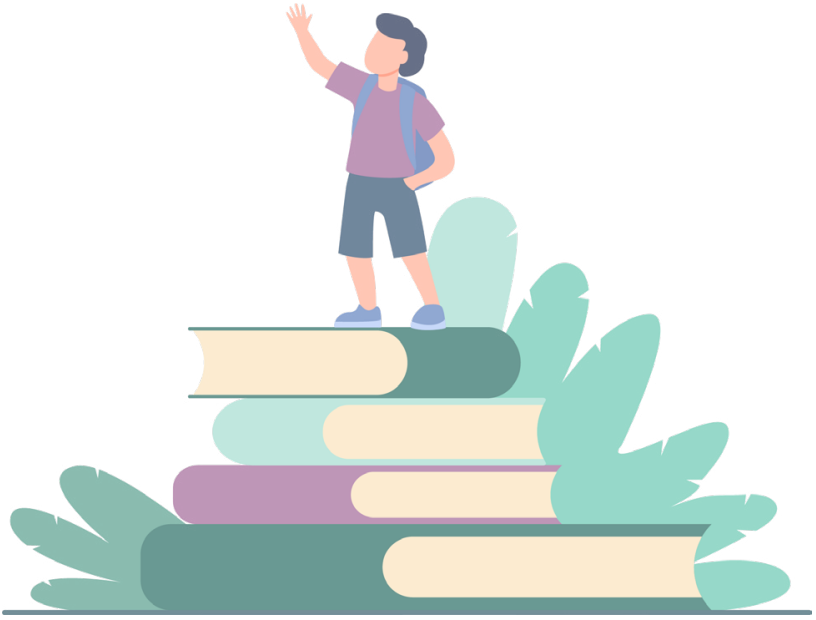




\section{Introducción}

Este proyecto se basa en una observación orientada según la etnografía virtual [1]. Para ello nos basamos en Mosquera Villegas (2008):

No es de extrañar entonces que, así como han surgido en el transcurso del tiempo etnografías con nuevos apellidos, como etnografías antropológicas, científicas o educativa, hoy se nos presenten nuevas etnografías relacionadas con las nuevas tecnologías con las que se construyen nuevas realidades en la vida cotidiana (p. 538-539).

Durante el primer mes de cuarentena en Colombia, estuvimos observando las maneras como estudiantes, docentes y padres de familia se acomodaban a las nuevas realidades en lo referido al proceso formativo.

En abril de 2020, la mayoría de gobiernos del mundo estaban tomando medidas para evitar que el Covid 19, se siguiera expandiendo por cada rincón del planeta. Particularmente, el gobierno colombiano implementó distintas cuarentenas como medida obligatoria para combatir la propagación del virus, si bien las formas de implementación, los sectores y los planes de choque implantados no fueron recibidos con beneplácito en muchos sectores sociales. Esto se sentía mucho en las interacciones digitales (encuentros sincrónicos de clase, reuniones de docentes, grupos de WhatsApp) y fue crucial en la primera parte del trabajo de campo: estudiantes, padres de familia y docentes manifestaban todo tipo de quejas e inconformidades en medio de un ambiente agitado de incertidumbre.

En efecto, al restringir la movilidad y alterar las formas convencionales de reunión social, esta problemática afectó diversos sectores sociales, particularmente al sector educativo (De Vincenzi, 2020). Estudiantes de todos los grados educativos, desde primaria hasta educación superior, se vieron obligados a abandonar sus aulas de clase y quedarse en casa para evitar el contagio del virus. Para garantizarles el derecho a la educación, el Ministerio de Educación Nacional (MEN) propuso que instituciones, profesores y estudiantes acudieran a las tecnologías de información y comunicación (TIC) para solventar la dificultad de movilidad.

Pronto notamos que las decisiones tomadas con miras a la salud pública pusieron en evidencia bastantes asuntos referidos a la brecha digital, al implantar como medida obligatoria la migración de los procesos educativos a otros entornos educativos. De hecho, las formas de implementación que emplearon instituciones — públicas y privadas, en todos los niveles- dejó el serio cuestionamiento de qué tanta apropiación de las TIC en educación existen. También alertó sobre serias confusiones terminológicas en conceptos como "virtual", "digital" y "electrónico". Esto, visto a la luz de las reacciones de padres, estudiantes y docentes que veníamos observando, generó profundas inconformidades que se han vivido en distintos grados a través de los diferentes niveles educativos. 


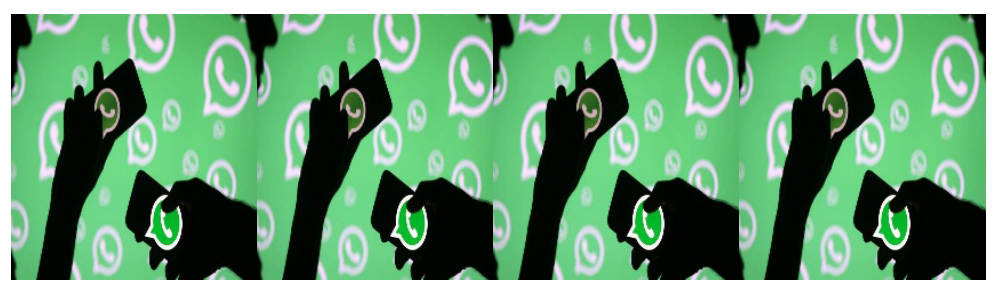

\section{Marco situacional}

El 12 de marzo de 2020 el Gobierno de Colombia emitió la Resolución 385, mediante la cual declaraba la "emergencia sanitaria por causa del Coronavirus" (Resolución Número 385 del 12 de marzo de 2020, 2020). Esta medida era la ratificación oficial de que el país también estaba siendo afectado por la situación que, a través de las noticias y las redes sociales, ya se conocía y se temía por parte de los ciudadanos. Así, el contexto emocional en el que se ingresó en la situación de excepción era bastante tenso, y los interrogantes sobre cómo se implantarían en cada sector las regulaciones aumentaron el clima de expectación.

Con el Decreto Legislativo 491, por el cual se adoptan “...medidas de urgencia..." a propósito de la situación, se estableció "...la modalidad de trabajo en casa, haciendo uso de las tecnologías de la información y las comunicaciones" (Decreto Legislativo Número 491 de 2020, 2020). Esta directriz fue expedida para instituciones públicas de educación, pero se tomaba como el parámetro que el Gobierno le daba al sector educativo en general. Sin embargo, aunque el texto no menciona "virtual" o "virtualidad" en lo referido a la educación [2], en el vocabulario de docentes, directivos, estudiantes y padres de familia, expresiones como educación virtual o virtualidad hacían parte constante de sus manifestaciones en relación con la situación.

Más allá de las diferencias terminológicas, pronto las dificultades comenzaron a surgir y a manifestarse. Era posible esperar que el cambio de modalidad educativa explicara las resistencias que desde todos los sectores se hacían escuchar. Sin embargo, el $96 \%$ de municipios del país no podían implementar actividades virtuales en colegios oficiales, debido a la falta de conectividad y de recursos tecnológicos (Laboratorio de Economía de la Educación, 2020). En el mismo informe se señalaba como lo que a menudo se nombra como "virtual" no es, en la inmensa mayoría de los casos, procesos educativos en entornos virtuales, sino la traducción, empírica —un tanto torpe y reactiva- "de los procesos educativos presenciales siendo intermediados por herramientas tecnológicas".

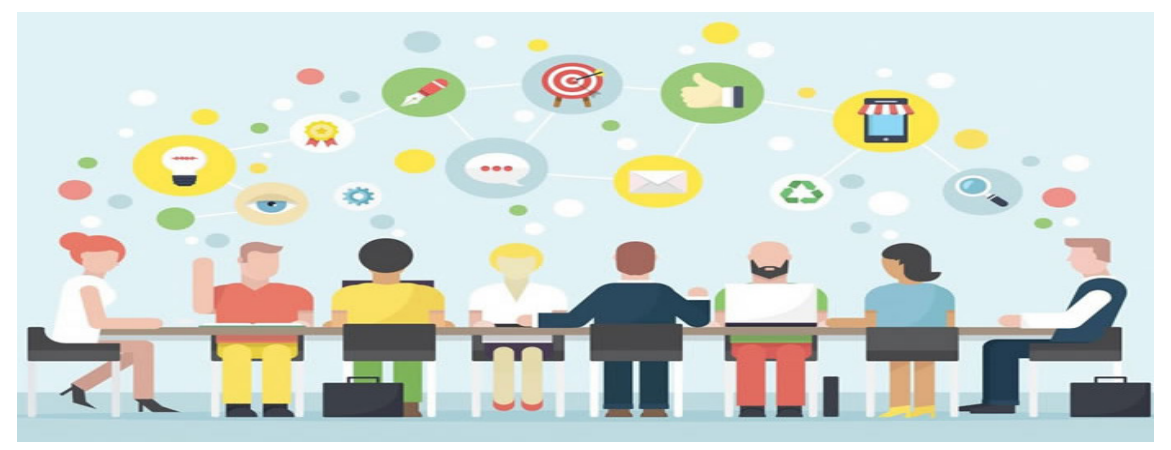

En un plano más amplio, esta situación coyuntural que se presentó dejaba la reflexión sobre la relación entre la educación y las TIC que, más allá de lo funcional que resultaba 
para efectos de una medida de "emergencia sanitaria", habla de cuán preparado está el país en términos sociales y técnicos para ingresar en los modos de producción, distribución y apropiación del conocimiento en el mundo actual. En el contexto de las sociedades del conocimiento, la brecha digital ha sido una problemática latente en Colombia, pese a las estrategias propuestas para contribuir a su reducción y a las políticas públicas que pretenden aumentar la infraestructura tecnológica y fortalecer la alfabetización digital (Gil et al., 2017). Así que ese cierto optimismo que se respira en las teorías de sociedad de la información y el conocimiento - donde las TIC son centrales - contrasta con los estudios y con las percepciones que se recogen en padres de familia, estudiantes y docentes.

Desde las primeras semanas en que se implantaron las medidas de educación en remoto, se evidenció en la reacción de estudiantes, padres de familia y profesores la enorme dificultad para migrar a entornos de aprendizaje mediados por las TIC. Más allá de las quejas, los señalamientos que se recogían permitieron anunciar algo más, una situación con un carácter mucho más profundo, estructural y sistemático (Quicios García et al., 2015; Vesga-Parra \& Hurtado-Herrera, 2013).

En otras palabras, algo que venía ganando terreno desde hacía mucho tiempo atrás y que el virus no hizo nada diferente que poner en primer plano, manifestado en los malestares individuales de los actores del proceso educativo y que se expresó de diversas formas como una inconformidad profunda. En otras palabras, si bien la situación pudiera tomarse como coyuntural, bajo los reclamos airados de los actores del proceso educativo se puso en evidencia una profunda dolencia social que, si bien pasa por lo específico de la conectividad (Gil-Juárez et al., 2015; Gil et al., 2017), el uso de los dispositivos (Sánchez et al., 2017) y el cambio repentino de modalidad (Ordorika, 2020; Reimers \& Schleicher, 2020), tiene que ver con la posibilidad de integrar esos modos de interacción en el mundo cotidiano, particularmente referido al aprendizaje (Bernal Acevedo, 2017).

Desde ahí, una primera constatación es cómo la brecha digital es un fenómeno que evidencia las enormes barreras (sociales, actitudinales, técnicas y simbólicas) que supone la asunción de la educación "virtual".

\subsection{Brecha ¿digital o social?}

La brecha digital se refiere a la diferenciación o separación producida entre individuos, grupos de individuos, instituciones, sociedades o países en el acceso y uso de las TIC. Puede ser definida en términos de la desigualdad de posibilidades que existen para acceder a la información, el conocimiento y la educación a través de la tecnología (Cabero Almenara, 2014, p. 15).

Las TIC constituyen una de las variables críticas de la sociedad del conocimiento, con un fuerte impacto en todos los sectores de esta sociedad, desde el económico al cultural, desde el ocio a la formación, y desde el cultural al de la comunicación (Guzmán, 2017). Estas tecnologías son fundamentales en el mundo de hoy. Pero la existencia de la brecha deja muchos interrogantes sobre las posibilidades reales de las TIC como promotores del cambio social (de la UNESCO, 2005). 
Esa "diferenciación" o "separación" de la que habla Cabero Almenara (2014) va desde lo terminológico hasta la posesión, uso y manejo de los dispositivos que permiten el acceso al mundo digital. Pero la confusión en la terminología evidenciada con motivo de la pandemia afecta su apropiación por parte de las personas.

En su aparición, el concepto "brecha digital" (digital divide), enfatizaba en las ventajas o desventajas que tendrían algunos sectores de la población según los grados de actualización de sus equipos tecnológicos. Como parte del gobierno Clinton, en Estados Unidos, la idea de "digital divide" se empleó en cuanto a lo administrativo para liderar políticas gestión de tecnología en los hogares (Cortés Vera, 2009).

Traducir digital divide a brecha digital implicó también acomodar el término a las particularidades de las poblaciones latinas. En este contexto, no preocupa tanto la actualización de los equipos, sino que se tengan los mismos para realizar diversas actividades (Flores Simental, 2008).

Siguiendo a Cabero Almenara (2014), se plantean entonces dos aspectos en los cuales se manifiesta la brecha: el técnico y el social. El primero se refiere a los dispositivos tecnológicos, mientras que el segundo enfatiza las formas como las personas incorporan los dispositivos a sus hábitos cotidianos.

Lo anterior se puede ver en que, pese a que algunas instituciones adquirieron licencias de plataformas y aplicativos educativos, o ampliaron las coberturas de otras que ya tenían, muchos factores impedían una migración adecuada a la denominada "virtualidad".

Debido a la confusión, desde el principio del trabajo de campo ya tuvimos claro que la brecha digital no era únicamente un asunto técnico. $\mathrm{Y}$ ni siquiera de acceso a las herramientas, sino una mezcla de temas, que captamos pronto en sondeos a padres, docentes y estudiantes de las instituciones. Desde las primeras observaciones, notamos descoordinación entre Gobierno, directivas y profesores, que llevaba a malos entendidos entre estos, sus alumnos y los padres de familia.

La suma de todos esos factores nos planteaba una diferencia entre la virtualidad de la que hablaban en todas partes y las formas como cada profesor ideaba para continuar adelante sus procesos de formación. Esta distancia se manifestaba de dos maneras. La distancia entre la ecuanimidad en el tono de los gobernantes cuando hacían sus alocuciones en televisión, que daba la sensación de que todo estaba bajo control, y el tono reactivo de los directivos de las instituciones, que dejaba ver claramente la confusión. Otra distancia estaba entre las directrices de los administrativos de los colegios y lo que los profesores lograban hacer, al conocer mejor a sus estudiantes y escuchar directamente todas las quejas de estos.

La diferencia entre la teoría y la práctica fue la que nos llevó a preguntarnos cuáles son las percepciones y dificultades que sobre la brecha digital expresan estudiantes, padres de familia y profesores de las instituciones estudiadas. 


\section{Metodología}

Para entender la etnografía nos basamos en Galindo Cáceres (1998) y Mosquera Villegas (2008). Para Galindo la etnografía propone "el oficio de la mirada y el sentido", es decir, recupera lo cotidiano como espacio de construcción de significado. Por su parte, Mosquera Villegas se apoya en varios autores y sintetiza las características de la etnografía digital en: participación intensiva del investigador en el medio investigado, enfoque exploratorio, tentativo y de apertura mental, y uso de técnicas múltiples, no siempre las convencionales de la investigación.

De Galindo retomamos el análisis detallado de los fenómenos, y de Mosquera Villegas, la inmersión de los investigadores en el ambiente estudiado mediante el uso de técnicas no convencionales que no hacen sentir como vigilados a los participantes.

Realizamos tres fases de observación. La primera (abril) sirvió para identificar las reacciones más álgidas de la comunidad educativa. En la segunda (mayo) profundizamos la observación en entornos educativos formales y no formales. En la tercera (junio) aplicamos las entrevistas. A cada uno de los grupos de estudio se les hizo una ronda de entrevistas semiestructuradas para reconocer sus actitudes, capacidades y percepciones en torno a la coyuntura que les tocó vivir y, de manera particular, a las formas como idearon para acomodarse a esa nueva situación. Cada observación fue registrada en una ficha, y el total de observaciones se reunió en una matriz de análisis con los registros de interacciones y entrevistas. Como apoyo, utilizamos una bitácora digital donde reunimos tomas de pantalla, audios, fragmentos de conversaciones y publicaciones digitales de los miembros de las comunidades (estudiantes, docentes y padres de familia).

Para el análisis nos basamos en los postulados de Galindo Cáceres (1998) y Chávez (Méndez, 2007) de buscar convergencias y divergencias en los datos hallados. Esto permitió encontrar puntos de encuentro comunes en las observaciones, así como puntos de menor importancia cuantitativa pero alta relevancia significativa desde el problema de investigación. El procedimiento de análisis fue inductivo: desde los registros obtenidos, fuimos elaborando la categorización en la que estructuramos la presentación de los resultados: acceso, uso y calidad. A partir de una serie de conceptos sobre brecha digital, se elaboraron fichas de análisis de contenido, con las cuales se revisó la información que surgía en los entornos analizados de las tres instituciones.

Las instituciones del estudio fueron: San José de las Vegas (privada, ubicada en la ciudad de Medellín, Antioquia); Institución Educativa Normal Superior de Abejorral (pública, ubicada en Abejorral, Antioquia) e Institución Educativa San Antonio (pública, situada en Rionegro, Antioquia). Los grados escolares respectivos, fueron: primero y quinto, para la primera institución, sexto, octavo y once, para la segunda, y primero a quinto en la tercera. La selección se hizo con base en el criterio de muestreo por conveniencia, al ser instituciones en las cuales los autores, se desempeñan laboralmente. Para ampliar el espectro de observación, se distribuyó las rutinas de trabajo de campo, de tal forma que además de la autora vinculada a la institución, otra la asistió en el proceso; estas miradas se pueden denominar auto-etnográfica y etnográfica propiamente dicha siguiendo los postulados de 
la etnografía y, en general, de la investigación cualitativa, se privilegió la diversidad de los datos, antes que su concentración, con el fin de acopiar la mayor variedad y diferencia entre los contextos de estudio.

\section{Resultados}

En esta investigación se tomaron en consideración las percepciones de estudiantes, docentes y padres de familia frente a la nueva realidad a la que Colombia se vio sometida sin mucha prelación en el aviso ni suficiente tiempo de preparación, debido al choque de la coyuntura- obligó cambios en la manera de enseñar, de acompañar en el proceso formativo y de aprender. Obligó a padres de familia, docentes y estudiantes a utilizar métodos diferentes a los que venían acostumbrados, cambiando aulas de clase, tableros, recreos en el patio, cuadernos, por reuniones que se llevan a cabo por medio de aplicaciones interactivas, pantallas de dispositivos electrónicos, videos explicativos, entre otros. Un choque de este tipo implica resistencias, nuevos aprendizajes y reacciones que nosotras leemos desde la percepción de los protagonistas.

\subsection{Estudiantes}

En las percepciones de estudiantes, la atención se centra en lo repentino de la medida, en cuanto esto les dificulta el aprendizaje por asuntos que no tienen que ver directamente con los temas tratados en clases y en cómo se pierde el componente de socialización que implicaba la educación presencial.

La situación de las TIC nos tomó por sorpresa, pues no estamos capacitados lo suficiente para manejar como se debe estas tecnologías que implican la manipulación constante de plataformas virtuales. Ha sido un dolor de cabeza para muchas familias y estudiantes, pues la situación económica no es la mejor y no cuentan con todas las herramientas necesarias para llevar a cabo dichos procesos de aprendizaje (EE4, Institución pública).

Yo me demoro mucho haciendo las guías, si tuviera computador o internet sería más fácil hacerlas, sobre todo cuando nos ponen a ver videos; pero como no tenemos, mi mamá va al colegio cada ocho días y las recoge impresas, pero me demoro en enviarlas a las profes, aunque algunas veces mamá compra datos y les puedo enviar fotos de las tareas. Si mamá no tiene datos toca llevar las guías al colegio para que las profes me las revisen (EE1, institución pública).

Cuando se da el caso de estudiantes que poseen los dispositivos requeridos, ocurre a veces que no ni poseen los conocimientos necesarios para hacer uso de estos: "Cuando estoy en el computador mirando las tareas, me meto a Classroom pero más que todo es cuando hacemos videollamadas se me bloquea" (EE2, Institución pública). Otro estudiante complementa este aspecto: “... a veces el internet no me da para ingresar a la plataforma, también cuando hay encuentro virtual con la profesora me salgo mucho de la llamada y la voz se escucha rara" (EE3, Institución pública). 
Se evidencia que algunos estudiantes tienen uso de algunas herramientas tecnológicas, pero la baja conexión no les permite participar activamente de las clases. Estos asuntos se mezclan con dificultades puntuales que se identificaron a lo largo de la observación: desatención, dificultad para comprender las explicaciones, problemas para reconocer los turnos de habla (cuándo apagar o prender el micrófono), seguir adecuadamente las indicaciones dadas por profesores para el envío de trabajos.

Ante esto, los estudiantes ingresan en otro tipo de relación con padres de familia y docentes: "Hay veces que mis papás no me pueden acompañar, porque mi papá trabaja y mi mamá tiene que hacer aseo en la casa" (EE2, Institución pública), lo cual genera interrogantes sobre esos ambientes emergentes que se forman entre las formas comunicativas y las competencias de los estudiantes, las estrategias que emplean los docentes, y las decisiones que, en casa, toman los padres de familia.

"Mi mamá me colabora en mis actividades y talleres, solo que ella le debe ayudar más a mi hermanita que está en preescolar, y algunas veces me toca esperar que ella se desocupe para preguntarle algo que no entiendo, o si no lo hago solo" (EE3, Institución pública) [3].

\subsection{Docentes}

En cuanto a los docentes, sus testimonios son significativos, aunque no tanto el poder acceder a los equipos sino en sacarle un debido provecho acorde a los propósitos educativos. De acuerdo a las entrevistas realizadas se encontraron diversidad de experiencias con el proceso de las clases virtuales:

Hay muchas dificultades con los datos para navegar en internet y así los datos que los estudiantes recargan se les acaba muy rápido, por lo que yo monto las planeaciones a la plataforma por instrucción de la institución, pero el archivo también lo montó al grupo del WhatsApp en PDF, como el PDF a muchos no les abre, les monto foticos de las actividades que hay que realizar, los vídeos se los bajo descargados porque eso posibilita que no gasten muchos datos, y en matemáticas no estoy enviando videos: compré un tablero y con el tablero les explico las cosas básicas. Los niños están muy animados, la recepción de las tareas ha sido a través de fotos (ED1, Institución pública).

Algo que se observa transversal en la investigación, es cómo los docentes se sienten en medio de dos situaciones opuestas, en tanto mediadores de las políticas macro del Gobierno, las directivas institucionales, el encuentro con los estudiantes y la relación con los padres de familia. Como aspecto notable, el ingreso de los padres a las clases generaba todo tipo de reacciones, desde las más positivas hasta las que reclamaban que esta injerencia podría ser negativa en ocasiones [4].

Esto de la virtualidad es una situación que nos cogió a todos sin herramientas suficientes para trabajar. Yo soy una persona que manejo poco la tecnología y me ha tocado sufrir mucho para manejar las herramientas tecnológicas, pero gracias a mi hijo que me ha colaborado mucho, he podido irme ubicando y así 
he podido trabajar con los niños, que por la cantidad tampoco ha sido fácil. Ahora estoy trabajando por WhatsApp y también de forma física, aunque de esta forma los padres de familia también han tenido muchas dificultades, poco a poco me he ido adaptando a este proceso (ED2, Institución pública).

De acuerdo con esto, no solo los docentes presentan grandes dificultades para el manejo de la tecnología, sino que deben aprender a distinguir otro tipo de conocimientos que ahora entran en el espacio formativo. En ocasiones se dificulta establecer un ritmo de clase adecuado y precisar cuándo alimentar esos otros asuntos alternativos a lo académico.

Las dificultades en la práctica docente son diversas y han cambiado a lo largo del proceso; entre las más relevantes para mí es la interacción permanente con los estudiantes, pues ésta en ocasiones presenta altibajos según los recursos de los estudiantes y las programaciones o plataformas que tengan las instituciones educativas. Otra dificultad es poder evidenciar de forma frecuente los saberes previos, ideas, concepciones y aprendizajes de los niños, pues este proceso de la virtualidad se reduce a algunas representaciones gráficas o interacciones virtuales (ED3, Institución pública).

Sin embargo, algo que permanece en todos los testimonios de profesores es la sobrecarga de trabajo.

En esta cuarentena me he sentido un poco abrumada con tantas cosas por hacer al mismo tiempo, desempeñamos muchos roles y yo trato de hacerlos de la mejor forma posible, sin embargo, no dejo de sentirme cansada y agobiada, con el manejo de las herramientas tecnológicas que han dispuesto en mi colegio para las clases virtuales, pues yo nunca había dado una clase virtual y poco a poco he ido aprendiendo, es un aprendizaje continuo para los docentes, los estudiantes y las familias, aun así no podemos pretender pasar o trasladar el colegio a las casa, por lo que debemos priorizar los contenidos para cada grado y enseñar lo esencial (ED4, Institución privada).

Así, la labor de los docentes se ha convertido en un espacio de laboratorio, experimentación y recursividad. Si bien al comienzo de la cuarentena se notaban altos niveles de saturación y desgaste, la migración a otras modalidades de aprendizaje "...ha permitido aprender y descubrir las bondades que tienen las herramientas tecnológicas, ha permitido clases más dinámicas en los estudiantes. Me ha llevado a ser recursiva, creativa y a enfrentarme sí o sí a realidades a las cuales le temía" (ED5, Institución privada).

Este asunto, podría aumentarse con la debida capacitación. Sin embargo, la falta de ésta frente al manejo de las herramientas tecnológicas ha sido un obstáculo significativo para que los estudiantes logren alcanzar las competencias esperadas: "Para mí lo más difícil ha sido la conectividad, sé muy poco de tecnología y por eso ha sido difícil comunicarse con los niños y padres de familia, solo lo hago por WhatsApp. Me ha costado mucho adaptarme a este nuevo modelo de enseñanza" (ED6, Institución pública). 
Se puede evidenciar entonces cómo en las instituciones es una constante el aprendizaje continuo que han tenido que tener los docentes para tratar de hacer las cosas de la mejor forma posible.

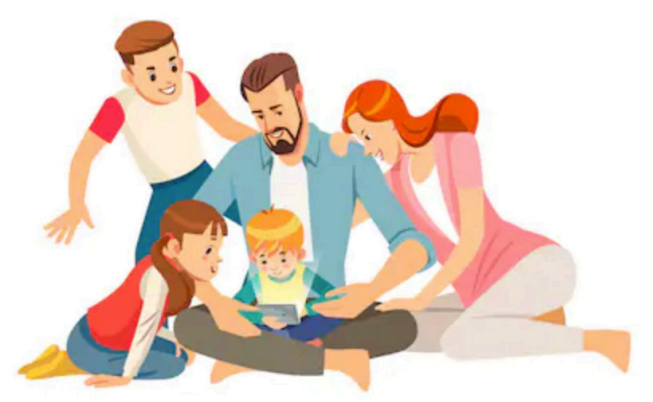

\subsection{Padres de familia}

Según los datos recogidos, es común encontrar que para algunos padres de familia el no tener los dispositivos adecuados les impide darle un buen uso a las plataformas digitales que han sido designadas por los maestros para el buen rendimiento académico y se han visto sumergidos en el deber de aprender a manejarlas y reconocer para qué sirve cada una: "Lo que estamos viviendo ahora es educación de contingencia, es decir, ni padres-madres, ni maestros, ni estudiantes estábamos preparados para realizar los procesos educativos a través de herramientas virtuales. Por eso es preciso reconocer que estamos aprendiendo" (EP1, Institución privada).

Por lo anterior, y debido a lo imprevisible de esta contingencia, lo que se ha evidenciado es que los recursos tecnológicos, aunque se tengan, no garantizan por sí mismos el uso de la tecnología para el aprendizaje:

Como padres de familia nos ha parecido un cambio muy brusco ya que no todos los alumnos cuentan con un computador y solo manejan el celular, de igual manera los profesores no se organizan y creen que como los niños están en casa los pueden saturar y saturar a los alumnos de tareas (EP2, Institución pública).

Esto se ha traducido en que los padres de familia y los estudiantes están sintiendo una carga académica bastante pesada, donde deben emplear más tiempo y esfuerzo al que estaban acostumbrados cuando asistían a las clases presenciales.

Esto de lo virtual es muy agotador, la niña se sienta en el computador desde las $6.30 \mathrm{am}$, hasta las $3.00 \mathrm{pm}$ [lo cual] es complicado porque son muchas clases que les dejan, son temas y talleres en el link y deben desarrollarlos y subirlos a un muro de la plataforma. La clase se interrumpe mucho porque hay niños muy indisciplinados y abren los micrófonos interrumpen o por chat escribiendo muchas cosas (EP3, Institución privada).

Algunos padres de familia incluso han juzgado el hecho de que sienten que le están haciendo el trabajo a los maestros: 
Como familia que acompaña el proceso de mis hijos me parece bien complicado no solo por la virtualidad, sino por la falta de explicación por parte de los docentes, ya que muchos solo se dedican a enviar talleres y nosotros somos los que debemos mirar cómo explicar las actividades a los muchachos (EP4, Institución pública).

Una madre de familia, con una situación similar a la anterior, opinó:

Cuando están en clase en línea y los profesores están explicando algún tema, se da cuenta que son pocos dinámicos a la hora de explicar un tema y lo único que hacen durante la hora de clase es hablar y hablar y poca participación de los estudiantes y se ven pocos videos, imágenes, etcétera, en la explicación. Además de eso son algo injustos porque los estudiantes pueden estar en clase y de pronto se cae la clase, o se quedan sin Internet. No explican bien los temas, quedan muchas dudas en los estudiantes a la hora de hacer los trabajos, ponen muchas tareas. Se exageran con los trabajos (EP5, Institución pública).

Lo que más resalta en esta diversidad de percepciones es la incomodidad a la que estudiantes, docentes y padres de familia se ven obligados. Una situación en la que nadie puede darles respuestas consistentes, y en la que las dudas crecieron día a día en medio de un clima mundial agitado por las noticias de la expansión del virus.

\subsection{Discusión}

Hemos acopiado los puntos más importantes de las percepciones de las comunidades educativas en las instituciones mencionadas y las hemos relacionado con lo que nos deja la observación orientada por la etnografía digital. El punto siguiente es discutir cómo esos resultados nos hablan de la brecha digital. Para ilustrarlo con claridad, categorizamos las convergencias en los datos de acuerdo a los aspectos de brecha digital que en cada caso se ponían de relieve.

Por lo anterior, clasificamos los resultados desde el acceso, el uso y la calidad. Nos basamos en Martínez Domínguez (2020), quien clasifica la brecha digital en conectividad, usos y tipos de usos. Sin embargo, la "conectividad" en nuestra investigación no es un término adecuado, porque para que la conexión sea posible, la posesión del dispositivo es indispensable. Por eso reemplazamos conectividad por acceso, pues vimos que el acceso no estaba garantizado por la posesión del dispositivo.

En la siguiente tabla sintetizamos el cuadro de resultados clasificados en la discusión en términos de acceso, uso y calidad. 
Tabla 1.

Percepciones y dificultades de la brecha digital

\begin{tabular}{|c|c|c|c|}
\hline & $\begin{array}{l}\text { ACCESO (equipos, } \\
\text { conexión) }\end{array}$ & USO (plataformas) & $\begin{array}{l}\text { CALIDAD } \\
\text { (ambientes) }\end{array}$ \\
\hline Percepciones & $\begin{array}{l}\text { Estudiantes } \\
\text { Sin los recursos } \\
\text { mínimos no puedo } \\
\text { acceder a mi proceso } \\
\text { educativo } \\
\text { Docentes } \\
\text { No es fácil ingeniar } \\
\text { estrategias para los } \\
\text { estudiantes que no } \\
\text { tienen acceso }\end{array}$ & $\begin{array}{l}\text { Estudiantes } \\
\text { No sé utilizar las plataformas } \\
\text { No estamos preparados para } \\
\text { un proceso educativo en estas } \\
\text { condiciones } \\
\text { Docentes } \\
\text { No sé darles un uso educativo a } \\
\text { las plataformas } \\
\text { No sé plantear protocolos de } \\
\text { interacción en clase } \\
\text { Debo resolver mis propias } \\
\text { dificultades y tener soluciones } \\
\text { para los estudiantes } \\
\text { Padres de familia } \\
\text { No tengo facilidad en acompañar } \\
\text { a mis hijos en el uso de algunas } \\
\text { plataformas }\end{array}$ & $\begin{array}{l}\text { Estudiantes } \\
\text { La clase es aburrida } \\
\text { Me distraigo fácil } \\
\text { Docentes } \\
\text { Es difícil mantener la } \\
\text { atención } \\
\text { Los padres de familia no } \\
\text { entienden lo difícil de esta } \\
\text { labor en esta modalidad } \\
\text { Los estudiantes sacan } \\
\text { excusas más fáciles } \\
\text { ahora } \\
\text { Padres de familia } \\
\text { Mis hijos no están } \\
\text { aprendiendo igual } \\
\text { La "virtualidad" debería } \\
\text { reducir los costos de la } \\
\text { educación }\end{array}$ \\
\hline
\end{tabular}




\begin{tabular}{|c|c|c|c|}
\hline & $\begin{array}{l}\text { ACCESO (equipos, } \\
\text { conexión) }\end{array}$ & USO (plataformas) & $\begin{array}{l}\text { CALIDAD } \\
\text { (ambientes) }\end{array}$ \\
\hline Dificultades & $\begin{array}{l}\text { Equipos } \\
\text { Disponibilidad de los } \\
\text { equipos } \\
\text { Posesión de los } \\
\text { dispositivos } \\
\text { Actualización de los } \\
\text { equipos } \\
\text { Conexión } \\
\text { Acceso a internet } \\
\text { Calidad de la conexión } \\
\text { (estabilidad, velocidad) }\end{array}$ & $\begin{array}{l}\text { Plataformas } \\
\text { Hay usos que el móvil no logra } \\
\text { cubrir } \\
\text { Generación de contenido "para" } \\
\text { plataformas educativas } \\
\text { Contenidos } \\
\text { Inadecuación entre los } \\
\text { contenidos y las didácticas } \\
\text { empleadas en el entorno digital } \\
\text { Interacciones } \\
\text { Turnos de activación/ } \\
\text { desactivación del micrófono }\end{array}$ & $\begin{array}{l}\text { Lo educativo } \\
\text { Agotamiento en docentes } \\
\text { y estudiantes } \\
\text { Dificultad para generar } \\
\text { ambiente académico } \\
\text { Lo social } \\
\text { Dificultad para mediar } \\
\text { espacios sociales en } \\
\text { medio de la clase } \\
\text { Competencias digitales } \\
\text { Se mantiene la } \\
\text { traducción de las formas } \\
\text { convencionales a los } \\
\text { entornos mediados por } \\
\text { las TIC }\end{array}$ \\
\hline $\begin{array}{l}\text { Soluciones } \\
\text { alternativas } \\
\text { registradas }\end{array}$ & $\begin{array}{l}\text {-Repartir los tiempos } \\
\text { de uso de los equipos } \\
\text { disponibles en familia } \\
\text {-Tareas en equipo y/o } \\
\text { familia }\end{array}$ & $\begin{array}{l}\text {-Fotocopias, trabajos manuales } \\
\text { presentados en físico } \\
\text {-WhatsApp }\end{array}$ & $\begin{array}{l}\text {-Videos de apoyo } \\
\text {-Inmersión activa del } \\
\text { entorno familiar }\end{array}$ \\
\hline Autores & $\begin{array}{l}\text { Guzmán, } 2017 \\
\text { Gil et al, } 2017 \\
\text { Cao y Vaca,2018 } \\
\text { Sánchez, L., Reyes, A. } \\
\text { M., Ortiz, D., \& Olarte, F. } \\
\text { (2017). }\end{array}$ & $\begin{array}{l}\text { UNESCO, } 2005 \\
\text { Mur Sangra, } 2016 \\
\text { Baca-Pumarejo et al, } 2018 . \\
\text { Juste, et al, } 2015 .\end{array}$ & $\begin{array}{l}\text { Mendoza-Ruano y } \\
\text { Caldera-Serrano (2014) } \\
\text { De Vincenzi, A. (2020) } \\
\text { García, et al (2020) }\end{array}$ \\
\hline
\end{tabular}

\subsection{Acceso}

En el cambio de digital divide a brecha digital se evidencia la importancia del acceso a las TIC (Cortés Vera, 2009). Si bien resulta obvio que la falta de acceso constituye la brecha digital, este primer nivel habla, en el caso puntual de Latinoamérica, y como pudimos comprobar en la investigación, de las dificultades de acceso a los dispositivos y a la conectividad pues "se percibe que estas tecnologías van a producir diferencias en las oportunidades de desarrollo de las poblaciones y que se establecerá una distancia entre aquellas que tienen o no tienen acceso a las mismas" (Mendoza-Ruano \& Caldera-Serrano, 2014). En nuestra investigación, esta dificultad se aprecia más en las instituciones públicas. 
En los estudiantes, las percepciones de acceso apuntan al sentirse aislados del proceso educativo por no contar con los equipos o las conexiones a internet. Llama la atención que el dispositivo por excelencia es el teléfono móvil (Quicios García et al., 2015) y la aplicación más usada es WhatsApp (García et al., 2020). Si bien estos no han sido diseñados con propósitos educativos, han permitido, en medio de la circunstancia, el acceso.

En los docentes, el acceso no alcanza a verse como dificultad, pues la mayoría tienen equipos de cómputo y teléfono móvil, con lo cual solventan el mínimo de actividades que les permite ejercer su rol.

En cuanto a los padres de familia, las percepciones apuntan a la dificultad que supone garantizar para todos sus hijos los equipos y las conexiones a internet.

La observación registra un alto número de dificultades en este nivel de acceso, que se manifiestan sobre todo en estudiantes y padres de familia de las instituciones públicas.

Como soluciones al acceso, identificamos todo tipo de estrategias: fotocopias y actividades realizadas en físico, distribuidas de manera ingeniosa por docentes, que a veces los estudiantes devuelven digital o físicamente; estudiantes que realizan actividades con otros compañeros de clase, o se apoyan en estos para el envío de las mismas; padres de familia que distribuyen los tiempos de acceso a los dispositivos familiares, acorde a las necesidades y tratando de cubrir la mayor cantidad de huecos posibles[5].

Estos procedimientos se aproximan a lo que expresan autores como Mendoza-Ruano y Caldera-Serrano (2014), Gil-Juárez, Feliu y Calsamiglia (2016) y Morales-Romo (2017), Ramírez-Castañeda y Sepúlveda-López (2018): el terreno del acceso a las tecnologías es un espacio de luchas políticas y sociales. Al respecto nos parece valioso reconocer en las tácticas ingeniosas de estudiantes, padres de familia y docentes una pugna por no quedar aislados de la brecha. Por eso reafirmamos algo que ya se dijo en este trabajo: que es difícil separar cuándo la brecha digital es "simplemente" un asunto técnico y cuándo es un asunto social.

En efecto, como lo expresan Hargittai y Hsieh (2013 citados por Baca, Pumarejo et al., 2018) la desigualdad digital es una forma de desigualdad social, donde las diferencias en el acceso a la tecnología tienen consecuencias para los derechos humanos, sociales y capital financiero. Por esta razón, en el contexto internacional el término tomó un significado diferente, fruto del conjunto de desigualdades en la distribución de la riqueza y de los indicadores de desarrollo humano que origina una segmentación más entre "info ricos" e "info pobres":

La fractura fundamental de las sociedades ricas avanzadas ya no viene determinada únicamente por el acceso a la propiedad de los medios de producción, ni siquiera por el factor de división en grupos de estatus determinado por las diferencias en el consumo. El acceso a la Información, y a través de ella al conocimiento, condiciona hoy en mayor medida la división y la estratificación social. De ahí que hoy hablemos, también, de inforicos e infopobres como categorías sociológicas reales (Baigorri, 1998, p. 1). 
Lo que muestran estos aspectos es que la brecha digital es una traducción a los entornos tecnológicos de las brechas sociales que está previamente marcadas en las respectivas comunidades. Según varios autores, esta sería una característica latinoamericana (BacaPumarejo et al, 2018), aunque se vive con particularidades en Colombia:

Vemos cómo docentes y estudiantes de las escuelas públicas colombianas se encuentran hoy en la brecha digital; la falta de acceso por extensión y por profundidad invade las realidades de la escuela. Aunque existen programas nacionales encargados de dotar de infraestructura tecnológica y de alfabetizar digitalmente a docentes y directivos, estos no son suficientes frente a la problemática y las situaciones que enfrentan los propios actores escolares. La poca familiaridad que tienen los maestros y maestras frente al uso de las TIC los lleva a no alcanzar procesos de apropiación personal ni profesional; tal situación se refleja al momento de decidir para qué y cómo usan las TIC en su cotidianidad y en sus prácticas pedagógicas. Las TIC se han domesticado en las aulas de clase y se orientan sin una propuesta de educación en medios, reduciéndose a un plano instrumental (Vesga-Parra \& Hurtado-Herrera, 2013, p. 148).

Esto implica un reto para la educación (Mur Sangrá, 2016). Si el acceso es la posibilidad de ingresar al mundo de las TIC, el constante acceso marca la exposición, y la exposición, como veremos en el próximo apartado, facilita la familiaridad. El punto delicado que analizamos aquí es cómo la mera posesión de equipos y conexiones no garantiza nada por sí mismo. Por eso, conforme a Mur Sangrá (2016), analizamos el siguiente nivel de dificultad: el uso.

\subsection{Uso}

Contar con los dispositivos y las conexiones no es suficiente para vencer la brecha digital. Para Acevedo, Toctaguano y Troya, el servicio de internet “...ha permitido desarrollar rápidamente la forma en que se ejecutan actividades sociales y económicas al acelerar el intercambio de información. Esta capacidad de descentralizar procesos facilita la optimización de actividades que generan oportunidades de eficiencia, productividad y efectividad" (Acevedo et al., 20192). Esto significa que sin competencias de uso la posibilidad de participar activamente en esa red de intercambio (para propósitos de aprendizaje, en este caso) la brecha se mantiene, aunque se tengan los recursos técnicos. Sin embargo, para investigadores como Pinto Arboleda, existe un:

[...] carácter homogenizador del concepto de brecha digital [que] se legitima en los documentos [como] una suerte de metonimia de la parte por el todo donde la brecha de tipo digital se utiliza para definir diferentes problemáticas que responden mayormente a temas relacionados con una brecha de tipo cognitivo (Pinto-Arboleda, 2012, p. 107).

En el "uso" hablamos de esas dificultades. Desde las percepciones de estudiantes, vimos cómo el sentir que no saben utilizar las plataformas se une a una queja por no estar 
preparados para un proceso educativo en estas condiciones. El uso entonces se refiere a saber cómo poner las herramientas a funcionar hacia un objetivo, que en este caso es el proceso de enseñanza-aprendizaje.

En las percepciones de los docentes, se registró constantemente el no saber cómo darles un uso educativo a las plataformas, cómo plantear protocolos de interacción en clase. Todo esto se resume en la sensación que tienen los docentes en cuanto deben resolver sus propios problemas además de asesorar a sus estudiantes (y padres de familia) en este mismo asunto.

Por su parte, los padres de familia perciben que deben orientar a sus hijos en el contenido de las actividades y lecciones, a la vez que en el manejo de los dispositivos en los que deben realizar las actividades y enviarlas.

El conjunto de dificultades identificadas en la observación lleva la brecha a un nivel que trasciende el acceso. Se trata de dificultades asociadas a las plataformas, los contenidos y las interacciones, como ya se vio en las percepciones antes mencionadas.

El uso es la posibilidad de emplear el dispositivo y la conexión en función de un propósito educativo. De esta manera, el uso empieza a tener dificultades cuando las posiblidades tecnológicas del teléfono móvil no permiten una fácil navegación por las plataformas educativas. Por esta razón, pese a que las instituciones estimularon el uso de plataformas como Classroom o Moodle, la falta de capacitación a los docentes y la dificultad de acceso a estas desde el teléfono móvil, hizo que WhatssApp se convirtiera en el estándar. A veces, los docentes montaron sus contenidos en plataforma, pero las actividades se distribuían por WhastApp. Aunque por directriz de algunas instituciones se pidió a los docentes que el envío de los talleres fuera por medio de la plataforma, en la gran mayoría de los casos (salvo en la institución privada) esto se tuvo que cancelar.

Se aprecia entonces dificultad en generar material para la plataforma por el lado de los docentes, dificultades en los estudiantes para navegarlo y dificultades en los padres de familia para acompañar a sus hijos en esta labor.

En cuanto a las interacciones, sobre todo al inicio de la cuarentena se observaron dificultades en establecer los momentos propicios para activar o desactivar el micrófono. A menudo, cuando los turnos de habla se perdían, y cuando varios participantes del encuentro sincrónico trataban de hablar al mismo tiempo, los docentes se vieron en serios problemas para controlar esta situación.

Retomando las palabras de Pinto-Arboleda (2018), entender la brecha como asunto técnico impide ver las competencias que deben desarrollar los participantes del proceso. En este caso, padres, docentes y estudiantes manifestaron, y esto se pudo corroborar en las observaciones también, dificultades para usar las TIC en el contexto de lo educativo.

Llama la atención el uso de aplicaciones como WhatsApp e, incluso, la distribución de talleres en fotocopias que luego son enviados en fotografías digitales por correo o chat, 
son apropiaciones tecnológicas. Es decir, aunque se alejen de los niveles más altos de desempeño de las tecnologías educativas, son también formas de emplear la tecnología (el fotocopiado, la fotografía, el chat... son tecnologías) para los propósitos educativos. Pese a ello, docentes, estudiantes y padres de familia no lo perciben así.

La desigualdad en la disponibilidad de las TIC sugiere que, es recomendable que las propuestas formativas se generen a partir de las demandas específicas, que se formulen a través de un diálogo constructivo entre los maestros, directivos del plantel y los funcionarios educativos, con las familias, debido a que el cambio tecnológico influye de sobremanera en la inclusión y la exclusión social y digital, y de la manera en que se enfrente este reto, definirá la forma en que se reduce o se amplía la brecha digital entre personas y las comunidades (Baca, Pumarejo et al., 2018, p. 43).

Retomando a Mendoza-Ruano y Caldera-Serrano (2014), Ramírez-Castañeda y SepúlvedaLópez (2018), Gil-Juárez, Feliu y Calsamiglia (2016) y Morales-Romo (2017),

Queda claro que la brecha digital es un terreno que exige la recursividad de los usuarios que viven esa distancia como un reto que deben superar. "Los productos tecnológicos de información y comunicaciones (TIC) son vistos como herramientas con las cuales, a partir de su acceso y/o uso, se espera mejorar la calidad de vida de las personas y de las sociedades que conforman" (Ramírez-Castañeda y Sepúlveda-López, 2018, p. 2).

Así, esta investigación es consistente con los resultados de Cao y Vaca (2018): lo que se aprecia en el espacio digital es una extensión de desigualdades sociales de vieja data.

\subsection{Calidad}

Entendida como "distancia", la brecha digital habla de la lejanía de un grupo social frente a un estándar. Ese estándar es fácil de fijar en los dos niveles anteriores, porque tanto el "acceso" como el "uso" son fáciles de identificar. Pero la calidad habla de asuntos diferentes (Juste et al., 2015). Por esta entenderemos lo que Mendoza-Ruano y Caldera-Serrano (2014) entienden como el componente más alto identificado en la literatura sobre el tema (en el que pocos autores se detienen): "La capacidad adecuada para hacer que la información accesible en la red pueda ser convertida en 'conocimiento' por el usuario” (p. 128).

Se trata de un nivel complejo en el que es difícil establecer precisiones. La calidad de la experiencia en los entornos puede diferir por factores externos o internos. Por eso retomamos las percepciones consultadas en las entrevistas.

Para los estudiantes, las clases se convirtieron en aburridas y por ello se distraían fácil. Desde sus propias explicaciones, esto es porque las clases se hicieron monótonas. Los docentes manifestaron que es difícil mantener la atención de los estudiantes, en clases largas, con temas complejos y sin facilidad para buscar recursos de apoyo. Por su parte, padres de familia percibieron que la calidad de la educación había disminuido, y en varias ocasiones pidieron que bajo esta modalidad "virtual" las matrículas deberían reducir el costo. 
Frente a lo que dicen los estudiantes, los docentes consideran que ahora los estudiantes tienen más coartadas para sacar excusas (es difícil comprobar si en realidad su conexión se interrumpió o si realmente su teléfono móvil se descompuso). En cuanto a lo que dicen los padres, perciben que, por el contrario, su trabajo se ha intensificado.

En este aspecto puntual es donde encontramos mayores diferencias entre la institución privada y las instituciones públicas. La calidad del proceso educativo se mejora mucho cuando el estudiante puede contar con el apoyo de adultos en casa. Esto se presentó en mayor medida en las familias de los estudiantes de las instituciones públicas. Muchos de estos adultos habían perdido sus trabajos, en su mayoría trabajos que exigen presencia (y fuerza) física, es decir, el tipo de trabajos que no se podía realizar de manera remota. Por el contrario, los padres de la institución privada, con trabajos más dirigidos al conocimiento y a la gestión de información, pudieron en su mayoría realizar los trabajos desde casa, con lo cual no podían acompañar a sus hijos en la realización de sus tareas.

De esta manera, al cotejar las percepciones y las observaciones del grupo investigador, advertimos que lo educativo se afecta por el agotamiento que estudiantes y docentes manifiestan. Igualmente, por la dificultad para generar un ambiente propiamente académico, debido a la simultaneidad de labores que hay en las casas. En lo social, se evidencia una carencia de espacios para compartir otro tipo de experiencias adicionales a las académicas, y que hacen parte fundamental de la formación en espacios físicos. Finalmente, se evidencia en todos los agentes del proceso dificultades para acoger los ambientes mediados por TIC en sus potencialidades; los procesos educativos se tradujeron y se adaptaron a lo digital, pero no se puede hablar de un nivel de competencias digitales que permitan hablar de educación virtual.

\section{Conclusiones}

Son muchas las situaciones que se han generado a propósito de esta coyuntura. En lo referido a la educación, lo ocurrido hasta ahora puede entenderse como una prueba de fuego que mide cómo está preparado el país para el futuro. No porque la educación presencial deba desaparecer, sino porque el acceso, uso y calidad de las interacciones sociales mediadas por TIC suponen un requisito en el mundo actual.

Sin embargo, lo que se aprecia es que, más allá de que el país no se esperaba una situación de este nivel de complejidad e imprevisibilidad, las TIC siguen siendo vistas más en el plano personal y social que como potenciadoras del aprendizaje.

Si bien sobre brecha digital existen investigaciones, en lo particular de la educación, y puntualmente sobre los roles de estudiantes, docentes y padres de familia, lo que se ha publicado es poco. La mayoría de las publicaciones realizan estudios cuantitativos sobre el acceso a las TIC, revisan políticas públicas para gestionar el ingreso a internet y dispositivos, y analizan el impacto de diferentes planes y estrategias implementadas en diversos países. Aún quedan muchos vacíos de conocimiento por explorar en el tema y, particularmente, en Colombia. 
En el contexto de esas cifras más lo presentado en esta investigación, advierten que los retos son muchos. Por el lado de acceso, hay muchas zonas del país que ni siquiera tienen conexión a internet. Pero este estudio muestra que la recursividad aflora en escenarios de dificultad y que con un teléfono móvil un gran número de labores educativas se pueden solventar. En cuanto al uso, es claro que el factor decisivo son los docentes, a quienes se los debe capacitar en competencias digitales. En cuanto a la calidad, es evidente que no se puede tratar de capacitaciones coyunturales, pues lo que se requiere es incorporar las TIC no solo como traductores de las cosas que se hacen cotidianamente, sino como formas de acceder al mundo actual.

Por eso, no es conveniente, al menos para las instituciones de este estudio, hablar de ambientes virtuales de aprendizaje. Se trata de fusiones, ideas recursivas, adaptaciones y adopciones de tecnologías de manera mixta y un tanto confusa. De ahí que propongamos hablar de ambientes alternativos de aprendizaje mediados por TIC. Proponemos que, más que traducir la clase convencional a una videollamada, instituciones, docentes y padres de familia piensen en estos ambientes alternativos como una opción que se puede seguir explorando.

Finalmente, exponemos estas preguntas para ambientar los diseños didácticos y orientar a los padres de familia en su apoyo a los estudiantes:

¿Cómo debe ser un AAA?; ¿Qué didácticas se pueden aplicar en estos espacios?; ¿Qué características debe tener un AAA desde lo tecnológico?; ¿Cómo se puede medir el progreso de aprendizaje en un AAA?; ¿Cómo debe ser la comunicación entre estudiantes y docentes?; ¿Qué hacer con los estudiantes y maestros que no pueden acceder a las nuevas tecnologías?; ¿Cómo se puede integrar a los padres de familia de manera proactiva en estos AAA?

\section{Referencias}

Acevedo, D., Toctaguano, S., \& Troya, C. (2019). Impacto de Facebook en la promoción de la salud en Santo Domingo de los Tsáchilas-Ecuador. Práctica Familiar Rural, 4(2), 1-7. https:// doi.org/https://doi.org/10.23936/pfr.v4i2.74

Baca, Pumarejo, J. ., Villanueva-Hernández, V., Aguirre-Ramírez, H. G., \& Cantú-Cervantes, D. (2018). Brecha digital en alumnos del sistema de eduación primaria en Tamaulipas, México: un panorama del futuro capital humano del estado. Ciencia UAT, 13(1), 35-49.

Baigorri, A. (1998). Info-ricos e info-pobres (pp. 1-3). Diario Hoy. https://www.eweb.unex.es/ eweb/sociolog/BAIGORRI/papers/inforicos.pdf

Bernal Acevedo, D. (2017). TIC y educación. Creación de modos narrativos transmediáticos en procesos de aprendizaje. Revista Luciérnaga, 9(18), 52.60. https://doi.org/https://doi. 
Cabero Almenara, J. (2014). Reflexiones sobre la brecha digital y la educación: siguiendo el debate. Inmanencia, 4 (2), 14-26.

Cortés Vera, J. (2009). ¿ Qué es la brecha digital?: una introducción al nuevo rostro de la desigualdad. Investigación bibliotecológica, 23(48), 233-239.

de la UNESCO, I. M. (2005). Hacia las sociedades del conocimiento. Publicaciones Unesco. París.

De Vincenzi, A. (2020). Del aula presencial al aula virtual universitaria en contexto de pandemia de COVID-19. Avances de una experiencia universitaria en carreras presenciales adaptadas a la modalidad virtual. Debate universitario, 8(16), 67-71.

Decreto Legislativo Número 491 de 2020, (2020).

Flores Simental, R. (2008). ¿Qué es la brecha digital?: una introducción al nuevo rostro de la desigualdad. Universidad Autónoma de Ciudad Juárez.

Galindo Cáceres, J. (1998). Etnografía: el oficio de la mirada y el sentido. En J. Galindo Cáceres (Ed.), Técnicas de investigación en sociedad, cultura y comunicación (pp. 347-384). McGrawHill.

García, M. F., Espinosa, Y. C., Ferrer, J. C. R., \& Castillo, N. (2020). USO DE LA TECNOLOGÍA CELULAR Y EL WHATSAPP. EXPERIENCIA EDUCATIVA EN TIEMPOS DE PANDEMIA. XIII Jornada UVS.

Gil-Juárez, A., Feliu, J., Vall-Llovera, M., CAlsAMiGliA, A., \& Conesa, E. (2015). De la investigación mediante relatos de vida al teatro social: el caso de la brecha digital de género. Universitas psychologica, 14(5), 1583-1598.

Gil, H. A. P., Castro, K. A. C., \& Bermúdez, G. M. T. (2017). La brecha digital en Colombia: Un análisis de las políticas gubernamentales para su disminución. Redes De Ingeniería, 59-71.

Guzmán, A. (2017). Brecha digital de las herramientas web 2.0, entre los docentes de la Institución Educativa Agropecuaria de Fonseca. Télématique, 16(1).

Juste, M., Carballo, J., \& López, B. (2015). Las personas mayores y las TIC. Un compromiso para reducir la brecha digital. Pedagogía Social. Revista Interuniversitaria, 26, 337-359.

Laboratorio de Economía de la Educación. (2020). Educación virtual, ¿el desafío es sólo tecnológico? Pesquisa Javeriana.

Méndez, M. G. C. (2007). El grupo de discusión: una estrategia metodológịca útil para generar conocimiento reflexivo en la investigación social desde la perspectiva cualitativa. UCOL. 
Mendoza-Ruano, J.-J., \& Caldera-Serrano, J. (2014). Umbrales para la determinación de la brecha digital: comparativa entre regiones desarrolladas. Transinformação, 26(2), 125-132.

Mur Sangrá, L. (2016). La nueva brecha digital. El futuro de las nuevas tecnologías en Primaria desde la formación del profesorado. Revista electrónica interuniversitaria de formación del profesorado, 19(2).

Ordorika, I. (2020). Pandemia y educación superior. Revista de la Educación Superior, 49(194), 1-8.

Pinto-Arboleda, M. C. (2012). La construcción de la referencia en torno al concepto de brecha digital en España. Universidad Complutense de Madrid.

Quicios García, M. del P., Ortega Sánchez, I., \& Trillo Miravalles, M. P. (2015). Aprendizaje ubicuo de los nuevos aprendices y brecha digital formativa. Píxel-Bit. Revista de Medios y Educación, 46, 155-166.

Ramírez-Castañeda, Luz Arabany Sepúlveda-López, J. J. (2018). Brecha digital e inclusión digital: fenómenos socio-tecnológicos. Revista EIA, 15(30), 89-97.

Reimers, F., \& Schleicher, A. (2020). Un marco para guiar una respuesta educativa a la pandemia del 2020 del COVID-19. Obtenido de Organización de Estados Iberoamericano: https://www. oei. es/uploads/files/news/Science-Science-and University/1777/covid-19educion-oei-2020-espan-ol-11-4-20. pdf.

Romo, N. M. (2017). Las TIC y los escolares del medio rural, entre la brecha digital y la educación inclusiva. Bordón. Revista de pedagogía, 69(3), 41-56.

Sánchez, L., Reyes, A. M., Ortiz, D., \& Olarte, F. (2017). El rol de la infraestructura tecnológica en relación con la brecha digital y la alfabetización digital en 100 instituciones educativas de Colombia. Calidad en la Educación, 47, 112-144.

Resolución Número 385 del 12 de marzo de 2020, (2020).

Vesga-Parra, L. del S., \& Hurtado-Herrera, D. R. (2013). La brecha digital: representaciones sociales de docentes en una escuela marginal. Revista Latinoamericana de Ciencias Sociales, Niñez y Juventud, 11(1), 137-149.

Villegas, M. A. M. (2008). De la Etnografía antropológica a la Etnografía Virtual. Estudio de las relaciones sociales mediadas por Internet. Fermentum. Revista Venezolana de Sociología y Antropología, 18(53), 532-549 


\section{Notas}

[1]El trabajo se deriva de la investigación "Brecha digital: dificultades y capacidades de la educación en entornos virtuales de aprendizaje", desarrollada en la Universidad Católica de Oriente como parte del trabajo de Maestría en Educación.

[2]El Decreto menciona ocho veces la palabra "virtual", siempre para referirse a las "actuaciones administrativas" (Artículo 6) o a los "servicios de arbitraje y conciliación" (Artículo 10). No menciona educación.

[3] Observación del 29 de abril: "Algunas niñas están solas durante el momento de clase, se escucha la voz de algunos padres de familia, pero no apoyando a su hija, sino hablando de sus cosas personales. Otros, por el contrario, les colaboran, pero regañándolas porque hablan mucho o porque no cierran el micrófono y dándoles la instrucción de cada actividad a realizar (Institución privada, grado 1).

[4] Observación del 28 de abril: "La mayoría de padres de familia acompañan a sus hijas en este proceso académico que se les está dando desde la virtualidad, pero constantemente hay reclamos sobre el tiempo tan extenso que ellos deben dedicar a su acompañamiento y en ocasiones no pueden hacerlo por sus compromisos laborales".

[5]Observación del 7 de mayo: "Es muy difícil ayudarles a los niños, a veces solo tienen un solo dispositivo tecnológico en casa y no tienen conexión fija a internet".

\section{Para citar este artículo}

Arango, C., Castaño, D., Cuervo, K., Baena, Y. (2020). Brecha digital y Covid-19: percepciones y dificultades. Un estudio en tres instituciones educativas de Antioquia Revista Luciérnaga Comunicación. Vol. 12 Núm. 24. Pp:111-134. https://doi.org/:10.33571/revistaluciernaga.v12n24a7

OJS: https://revistas.elpoli.edu.co/index.php/luc/issue/archive

Link: https://www.politecnicojic.edu.co/index.php/revista-luciernaga 